tertiary rheumatology centre. The frequency, temporal occurrence and the localization of arthritis was recorded. In addition, we searched for potential differences in other IgAV features between patients with and without arthritis.

Results: During the 132-month observation period we identified 328 new IgAV cases $(59.5 \%$ males, median (IQR) age $64.3(45.1 ; 76.1)$ years). Forty-eight $(14.6 \%)$ patients developed arthritis. Arthritis was the first IgAV manifestation in $16(4.9 \%)$ patients. Arthritis was mono-, oligo- and poly- articular (involving up to 15 joints) in $13(4.0 \%), 25(7.6 \%)$ and $10(3.0 \%)$ patients, respectively. Arthritis was most common in wrists and ankles (each in 18 (37.5\%) patients); metacarpophalangeal joints and knees (each in 11 (22.9\%)); proximal interphalangeal joints $(9(18.8 \%))$; elbows $(8(16.7 \%))$ and metatarsophalangeal joints (4 $(8.3 \%))$. Clinical differences between IgAV patients with and without arthritis are presented in table 1. Patients with arthritis were significantly younger, more commonly developed gastrointestinal tract involvement compared to those without arthritis. Arthritis remitted in all with immunomodulatory treatment (given predominantly for necrotic skin purpura or visceral involvement). Follow up (FU) data accessible for $42 / 48(87.5 \%)$ patients with arthritis showed that IgAV relapsed in $10(23.8 \%)$ patients during a median (IQR) 24.5 (12.9; 40.7) month FU. Relapses were limited to skin and/or kidneys, there were no relapses of arthritis.

Table 1. Clinical characteristics of IgA vasculitis patients with and without arthritis

\begin{tabular}{lccc}
\hline Clinical characteristics & $\begin{array}{c}\text { Arthritis IgAV } \\
(48)\end{array}$ & $\begin{array}{c}\text { Non-arthritis IgAV } \\
(280)\end{array}$ & P value \\
\hline Male gender (\%) & 56.3 & 60.0 & 0.636 \\
Age (years)* & $49.9(36.1-66.9)$ & $65.4(48.4 ; 77.5)$ & $<0.001$ \\
Ever smoker (\%) & 50.0 & 43.6 & 0.435 \\
Prior infection (\%) & 43.8 & 30.4 & 0.094 \\
Generalized purpura & 50.0 & 50.0 & 1.0 \\
Skin necroses (\%) & 35.4 & 49.6 & 0.085 \\
Gl involvement (\%) & 47.9 & 25.0 & 0.002 \\
Renal involvement (\%) & 47.9 & 46.8 & 1.0 \\
Elevated serum IgA (\%) & $33.3(11 / 33)$ & $52.0(115 / 221)$ & 0.061
\end{tabular}

Legend: * median and IQR; GI gastrointestinal;

Conclusion: Arthritis in adult IgAV was frequently oligoarticular, involved large and small joints of both upper and lower extremities, and was not prone to chronic course and recurrence. In addition, we found an association between arthritis and gastrointestinal tract involvement.

Disclosure of Interests: None declared

DOI: 10.1136/annrheumdis-2021-eular.324

\section{POS0824 THE LONG-TERM CLINICAL COURSE OF MUSCULAR VASCULITIS DEPENDING ONTHE ANTI- NEUTROPHIL CYTOPLASMIC ANTIBODY STATUS: A RETROSPECTIVE OBSERVATIONAL STUDY}

T. Yoshida ${ }^{1}$, K. Nishimura' ${ }^{1}$, D. Waki ${ }^{1}$, K. Mizukawa $^{1}$, N. Tanaka ${ }^{1}$, H. Murabe ${ }^{1}$ T. Yokota ${ }^{1}{ }^{1}$ Kurashiki Central Hospital, Department of Endocrinology and Rheumatology, Kurashiki, Okayama, Japan

Background: Skeletal muscle is known as one of the organ involvements of primary systemic vasculitis. ${ }^{1,2}$ Muscle inflammation is detected by magnetic resonance imaging, and necrotizing vasculitis is proved by muscle biopsy. ${ }^{3}$ As with systemic vasculitis or single organ vasculitis, glucocorticoid (GC) and immunosuppressants are used in its treatment. ${ }^{4}$ There are not many reports about muscular vasculitis, and its long-term clinical course after initial treatment, including the rates of relapse and mortality, remains unclear.

Objectives: To identify the predictors of relapse and mortality in patients with muscular vasculitis, especially focusing on the status of anti-neutrophil cytoplasmic antibody (ANCA).

Methods: We retrospectively reviewed patients diagnosed with necrotizing vasculitis with muscle involvements in our hospital between 2004 and 2020 . In all cases, muscular vasculitis was identified by muscle biopsy or magnetic resonance imaging. To focus on the clinical features of muscular vasculitis, we excluded patients with such severe organ involvements as cardiovascular, abdominal, cerebral, severe renal, and severe pulmonary involvements. We compared the 5-year cumulative incidence of relapse, the overall survival rate, and the dose of GC over 5 years between the ANCA-positive and ANCA-negative groups. A relapse was defined as any new or worsened state of disease activity requiring an escalation of GC dose. Gray's method was used for assessing the cumulative incidence of relapse. The log-rank test was used for assessing overall survival. The Mann-Whitney $U$ test was used for assessing the dose of GC. The possible factors for relapse in 5 years in a univariate analysis were selected for a multivariate analysis using the Cox proportional hazards model.

Results: Forty-nine patients were enrolled. The median age of onset was 77 (6982 ) years and $71.4 \%$ were women. There were 30 ANCA-positive patients $(90.0 \%$ with anti-myeloperoxidase) and 19 ANCA-negative patients. The median age and the number of patients with renal involvements were higher in the ANCA-positive group than in the ANCA-negative group $(73.0 \pm 9.29$ years vs. $79.5 \pm 20.28$ years, $p=0.0062$ and $7 / 30[23.3 \%]$ vs. $0 / 19$ [0.0\%], $p=0.034$, respectively). The Birmingham Vasculitis Activity Score (ver. 3), the induction dose of GC, and the rate of immunosuppressants use were not significantly different between the two groups. During the observational period, 24 patients relapsed. The 5-year cumulative incidence of relapse was significantly higher in the ANCA-positive group than in the ANCA-negative group $(p=0.026)$ (Figure 1). The Cox proportiona hazards model revealed that the presence of ANCA was an independent risk factor for relapse (hazard ratio: $3.15 ; 95 \%$ confidence interval 1.06-9.38; $p=0.040$ ). During the observational period, 9 patients died ( 3 died from cancer, 1 from interstitial pneumonia, 1 from cerebral hemorrhage, 1 from infection, and 3 from unknown reasons). The ANCA-positive group exhibited a higher mortality rate than the ANCA-negative group without a statistical significance $(p=0.12)$. The 5-year cumulative dose of GC was larger in the ANCA-positive group than in the ANCA-negative group without a statistical significance (14786 [11246-19138] mg vs. 10088 [7129-12634] mg, $p=0.12$ ).

Conclusion: In muscular vasculitis, the presence of ANCA is an independent risk factor for long-term relapse. Stratified treatment depending on the ANCA status may reduce the relapse rate and the occurrence of side effects of GC in patients with muscular vasculitis.

\section{REFERENCES:}

[1] Kitching AR et al. Nat Rev Dis Primers 2020; 6(1): 71.

[2] Hernández-Rodríguez J et al. J Autoimmun 2014; 48-49: 84-9.

[3] Ushiyama S et al. Rheumatol Int 2020; 40(10): 1667-74.

[4] Ganeshanandan LR et al. Semin Arthritis Rheum 2020; 50(3): 503-8.

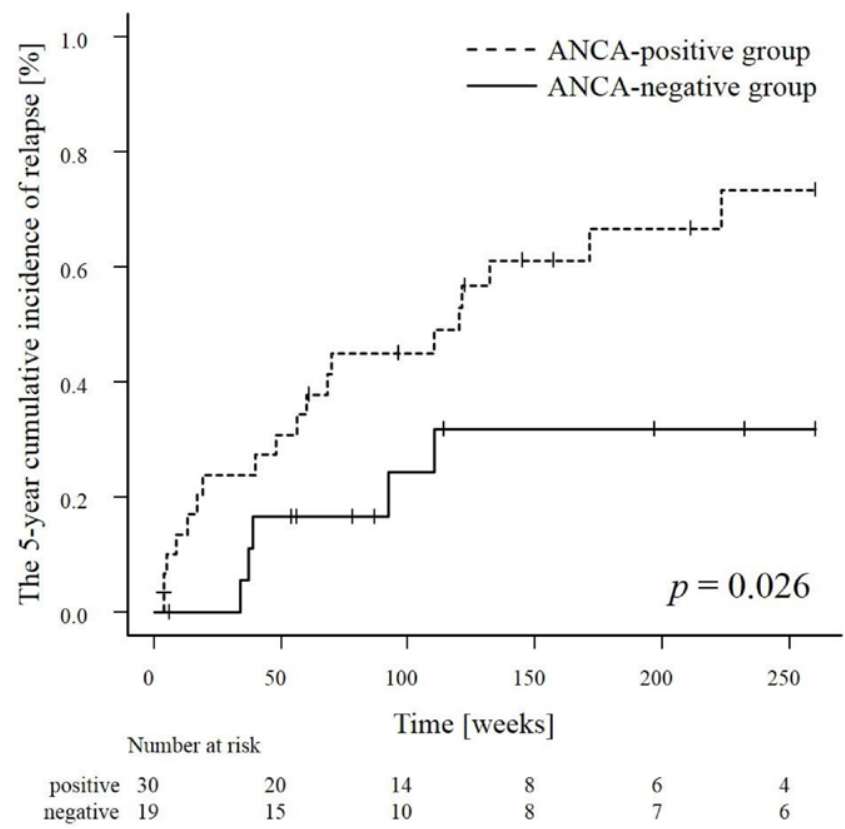

Disclosure of Interests: None declared DOI: 10.1136/annrheumdis-2021-eular.815

\section{POS0825 DEVELOPMENT OF A NEW AND SIMPLIFIED FORMULA TO PREDICT MORTALITY IN PATIENTS WITH ANCA-ASSOCIATED VASCULITIS ADMITTED TO THE INTENSIVE CARE UNIT}

P. Korsten ${ }^{1}$, F. Kück ${ }^{2}$, K. Tejiozem Donfack ${ }^{1}$, R. Vasko ${ }^{1}$, A. Lena ${ }^{2}$, B. Tampe ${ }^{1}$ ${ }^{1}$ Universitätsmedizin Göttingen, Department of Nephrology and Rheumatology, GÖTTINGEN, Germany; ${ }^{2}$ Universitätsmedizin Göttingen, Department of Medical Statistics, Göttingen, Germany

Background: ANCA-associated vasculitis (AAV) can present with a wide range of symptoms, including acute kidney injury (AKI) requiring renal replacement therapy or diffuse alveolar hemorrhage (1). These two manifestations often require admission to an intensive care unit (ICU) and are associated with increased mortality. To predict ICU mortality, the Simplified Acute Physiology Score version 2 (SAPS2) is often used but has not been formally tested in AAV patients (2). In addition, it is cumbersome to assess.

Objectives: To develop a novel, simplified formula to predict ICU mortality in an AAV ICU population from an academic tertiary care center. 